\title{
Moving Prevention Research Forward
}

\author{
Melissa F. Peskin
}

Published online: 6 August 2010

(C) Springer Science+Business Media, LLC 2010

It is a privilege to assume one of the Associate Editor positions for The Journal of Primary Prevention under the leadership of Dr. Susan Tortolero. I look forward to furthering the journal's mission to advance prevention research from a multidisciplinary perspective. The current issue represents this approach and includes a diverse collection of topics ranging from the role of peers in the development and prevention of adolescent risk behaviors to implementation, fidelity, and dissemination of effective programs. This compilation of articles represents JPP's commitment to "cutting edge" issues related to prevention research.

In the first article, Simons-Morton and Farhat provide a review of recent longitudinal research on social influence and adolescent smoking. Though researchers agree that peers strongly influence behavior, this review adds a unique perspective regarding the methods by which this influence occurs. Examples of methods include socialization and selection effects from peers and effects from parents in protecting youth against unhealthy peer influences.

Burns and colleagues also discuss peer influence but in the context of bullying behavior. They conducted a qualitative study and discuss the mechanisms by which

M. F. Peskin $(\bowtie)$

Health Promotion and Behavioral Sciences,

School of Public Health, The University of Texas,

Houston, TX, USA

e-mail: melissa.f.peskin@uth.tmc.edu peer groups can positively curtail bullying behavior. They suggest that bullying youth can be influenced to stop their negative behavior by prosocial friends who do not approve of this behavior and that youth can be positively influenced to leave a peer group that does approve of bullying.

In the studies led by both Simons-Morton and Burns, the authors provide important recommendations for how the positive aspects of peer influence (i.e., through friendships with peers who participate in prosocial and non-risky behaviors) can be incorporated into prevention efforts for changing social norms related to the prevention of adolescent risk behavior.

In the next three articles, the focus shifts to a different topical area, that of implementation and dissemination of effective interventions. As prevention researchers continue to develop and disseminate effective interventions, it is critical to understand the factors that accelerate their uptake and influence their implementation.

Shapiro and colleagues discuss the challenges and lessons learned in conducting widespread dissemination of the Triple $\mathrm{P}$ interventions, which are innovative parent-targeted strategies shown to reduce child maltreatment. Issues discussed include the time needed to coordinate large dissemination efforts and the need for methods to ensure program fidelity across sectors that provide a wide array of services. Many of these factors are applicable to the dissemination of other large-scale public health interventions. 
Kalichman and colleagues provide a closer look at program fidelity through their examination of the "operational" fidelity of the Healthy Relationships program, an intervention for individuals living with HIV/AIDS. This program has been extensively disseminated to multiple community agencies; however, it is not known to what extent the program's core elements are implemented. Interviews were conducted with agency contacts, and it was found that activities addressing the program's core elements were not always delivered as they were designed, most often to address a need for community fit. The authors note that agencies require more direction when adapting interventions and suggest that more studies are needed to determine how modifications affect "real world" program effectiveness.

Finally, Edberg and colleagues discuss the challenges related to the implementation of the SAFER Latinos project, a prevention initiative aimed at decreasing youth violence and developed using participatory methods. Although, as the authors suggest, great strides have been made in developing prevention initiatives for youth violence, this program is distinctive in that it has the potential to target community-wide factors. Strategies for overcoming implementation barriers, including many that focused around gaining community trust, as well as implications for evaluation and sustainability of such interventions are discussed. 